Article

\title{
Compositional Analysis of Whole Grains, Processed Grains, Grain Co-Products, and Other Carbohydrate Sources with Applicability to Pet Animal Nutrition
}

\author{
Alison N. Beloshapka ${ }^{1}$, Preston R. Buff ${ }^{2}$, George C. Fahey Jr. ${ }^{1}$ and Kelly S. Swanson ${ }^{1, *}$ \\ 1 Department of Animal Sciences and Division of Nutritional Sciences, University of Illinois_Urbana \\ Champaign, 1207 West Gregory Drive Urbana, IL 61801, USA; alison.beloshapka@gmail.com (A.N.B.); \\ gcfahey@illinois.edu (G.C.F.J.) \\ 2 The Nutro Company 1550 West McEwen Drive, Suite 100, Franklin, TN 37067, USA; Preston.Buff@effem.com \\ * Correspondence: ksswanso@illinois.edu; Tel.: +1-217-333-4189; Fax: +1-217-333-5044
}

Academic Editor: Anthony Fardet

Received: 20 December 2015; Accepted: 18 March 2016; Published: 25 March 2016

\begin{abstract}
Our objective was to measure the proximate, starch, amino acid, and mineral compositions of grains, grain co-products, and other carbohydrate sources with potential use in pet foods. Thirty-two samples from barley (barley flake, cut barley, ground pearled barley, malted barley, whole pearled barley, pearled barley flakes, and steamed rolled barley); oats (groats, ground oatmeal, ground steamed groats, instant oats, oat bran, oat fiber, oat flour, quick oats, regular rolled oats, steamed rolled oat groats, and steel cut groats); rice (brown rice, polished rice, defatted rice bran, and rice flour); and miscellaneous carbohydrate sources (canary grass seed, hulled millet, whole millet, quinoa, organic spelt hull pellets, potato flake, sorghum, whole wheat, and whole yellow corn) were analyzed. Crude protein, amino acid, fat, dietary fiber, resistant starch, and mineral concentrations were highly variable among the respective fractions (i.e., barley flake $v s$. malted barley $v s$. steamed rolled barley) as well as among the various grains (i.e., barley flake $v$ s. brown rice $v$ s. canary grass seed). These ingredients not only provide a readily available energy source, but also a source of dietary fiber, resistant starch, essential amino acids, and macrominerals for pet diets.
\end{abstract}

Keywords: amino acids; chemical composition; fiber; grains; pet food

\section{Introduction}

According to the American Association of Cereal Chemists (AACC), whole grains are defined as the intact, ground, cracked, or flaked caryopsis, which consists of starchy endosperm, germ, and bran portions that are similar to the intact caryopsis, and may be malted or sprouted, as long as labeled as such [1]. Whole cereal grains belong to the Poaceae or Gramineae families, better known as grasses. The endosperm is the largest portion of the grain and contains carbohydrates, proteins, vitamins, and minerals. The germ is also known as the embryo and contains vitamins, some protein, minerals, and fats. The bran portion is the outer covering of the grain, which protects the germ and endosperm. It contains phenolic compounds, vitamins, and minerals [2]. Whole grains, processed grains, and grain components vary greatly in their macronutrient and micronutrient composition and include a variety of bioactive compounds such as fiber, folate, phenolic compounds, lignans, and sterols [3]. Whole grains have been well studied for their application to human health $[2,4,5]$; however, the use of whole grains or grain fractions in pet food, specifically diets intended for canines and felines, has not been thoroughly evaluated despite how significantly cereal grains are used in today's pet food industry. These ingredients not only add nutritive value as readily available carbohydrate sources in diets for dogs or cats, but also contribute some essential vitamins, minerals, dietary fiber, fat, protein, and phytonutrients. 
The largest portion of a whole grain is comprised of starch, a polysaccharide composed of many glucose units, which is linked together with $\alpha-1,4$ and/or $\alpha-1,6$ glycosidic linkages [6]. Some of this starch may escape digestion in the small intestine, allowing for fermentation in the colon [7], and is commonly known as resistant starch (RS). There are four main types of RS: RS1 is made up of starch that is physically entrapped within a cellular or multi-cellular structure that prevents contact with digestive enzymes (e.g., a whole grain); RS2 is made up of raw starch granules with a crystal structure resistant to digestive enzymes (e.g., raw potato starch, green banana starch, and high amylose grains); RS3 is made up of retrograded starch that forms from repeated cooking and cooling (e.g., amylose that has recrystallized into a configuration highly resistant to digestive enzymes); and RS4 is made up of chemically modified starch, including starches that have been treated with chemicals to form ether or ester linkages with starch moieties, thus inhibiting their digestion by digestive enzymes [6].

Grains are a staple carbohydrate source in both human and pet diets. While canines and felines are classified as Carnivora, the modern dog in particular has evolved to consume a more omnivorous diet, and extruded diets remain the most common diet format fed to dogs and cats [8-10]. Carbohydrates in the form of cereal grains or alternative sources make up anywhere from $20 \%$ to $50 \%$ of most extruded diets [11]. In comparison to animal proteins or purified fiber sources, the low cost and high nutritive value of many cereal grains make them preferred ingredients for extruded pet foods. In general, worldwide cereal grain production ranks corn (maize) as the most widely grown with the highest production, followed by rice, which is a staple to over half of the world's population. Wheat has the third highest production, followed by barley, sorghum, and oats [12,13]. Cereal grains are widely used in the pet food industry, but poorly studied. Furthermore, many pet owners are apprehensive about feeding their pets cereal grains [14], resulting in a rise in the use of alternative carbohydrate sources, such as potatoes.

Extrusion, heat-treatment processing, and grain processing (i.e., fractionation) may alter the RS content, protein quality and digestibility, and bioactive compound concentration of a grain-containing ingredient. Previous research that compared common whole grains (barley, corn, oat, rice, and wheat) and their fractions before and after processing concluded that the RS concentrations of barley, corn, oat, and wheat were decreased after processing for $1 \mathrm{~h}$ at $100^{\circ} \mathrm{C}$ and expanded with hot air [15]. Unlike the cooking conditions used in that study which were processed in an extreme way to simulate extrusion, some whole grains are mildly processed prior to use (i.e., steamed, rolled, or kilned). Roasting (at $200{ }^{\circ} \mathrm{C}$ for $8 \mathrm{~min}$ ) and popping (at $250{ }^{\circ} \mathrm{C}$ for $90 \mathrm{~s}$ ) of grain amaranth have been observed to decrease protein digestibility when compared to raw samples of the grain [16]. Therefore, the objective of this study was to measure the proximate, starch, amino acid (AA), and mineral composition of various whole grains, processed grains, and grain co-products that may be incorporated into pet foods and treats.

\section{Materials and Methods}

\subsection{Samples}

Thirty-two samples were analyzed (one sample of each): barley samples (barley flake, cut barley, ground pearled barley, malted barley, whole pearled barley, pearled barley flakes, and steamed rolled barley; Supplemental Figure S1); oat samples (groats, ground oatmeal, ground steamed groats, instant oats, oat bran (from two different sources), oat fiber, oat flour, quick oats, regular rolled oats, steamed rolled oat groats, and steel cut groats; Supplemental Figure S2); rice samples (brown rice, polished rice, defatted rice bran, and rice flour; Supplemental Figure S3); and miscellaneous cereals and carbohydrate sources (canary grass seed, conventional hulled millet, conventional whole millet, conventional quinoa, organic spelt hull pellets, potato flakes, sorghum, whole wheat, and whole yellow corn; Supplemental Figure S4). 


\subsection{Chemical Analyses}

Grain samples were ground through a 1-mm screen in a Wiley mill (intermediate, Thomas Scientific, Swedesboro, NJ, USA). Duplicate samples were analyzed according to procedures by the Association of Official Analytical Chemists (AOAC) for dry matter (DM; $\left.105^{\circ} \mathrm{C}\right)$, organic matter $(\mathrm{OM})$, and ash (methods 934.01, 942.05) [17]. Crude protein (CP) content was calculated from Leco total $\mathrm{N}$ values (model FP-2000, Leco Corporation, St. Joseph, MI, USA; method 992.15) [17]. Total lipid content (acid hydrolyzed fat; AHF) of the samples was determined according to the methods of the American Association of Cereal Chemists (AACC) [18] and Budde [19]. Gross energy (GE) of the samples was measured using an oxygen bomb calorimeter (model 1261, Parr Instruments, Moline, IL, USA). Dietary fiber concentrations [total dietary fiber (TDF), soluble dietary fiber (SDF), and insoluble dietary fiber (IDF)] were determined according to Prosky et al. [20]. All samples were sent to the University of Missouri Experiment Station Chemical Laboratories for AA (method 982.30E) [17] and mineral analyses, including calcium (Ca; method 985.01) [17], chloride (Cl; method 943.01) [17], magnesium (Mg; method 985.01) [17], phosphorus (P; method 985.01) [17], potassium (K; method 956.01) [17], sodium (Na; method 956.01) [17], and sulfur (S; method 956.01) [17]. Compositional data were not analyzed using statistical methods because accuracy was ensured by adequate replication, with acceptance of mean values that were within $5 \%$ of each other.

\subsection{Starch Analyses}

Grain subsamples were ground through a $0.5-\mathrm{mm}$ screen in a Wiley mill (intermediate, Thomas Scientific, Swedesboro, NJ, USA). Values were determined in duplicate. The method of Muir and O'Dea [21,22] was used to determine the amount of starch digested in the stomach and small intestine by measuring glucose in the supernatant resulting from acid-enzyme digestion of the substrate. Briefly, $0.2 \mathrm{~g}$ of each substrate was weighed in duplicate and exposed to pepsin $/ \mathrm{HCl}$, amyloglucosidase, and $\alpha$-amylase. Tubes containing reagents but no substrate were run as blanks. All tubes were incubated for $15 \mathrm{~h}$ at $37^{\circ} \mathrm{C}$ and then centrifuged for $15 \mathrm{~min}$. Glucose concentrations in the supernatant were determined by reading the absorbance of individual samples at $450 \mathrm{~nm}$ on a DU 640 spectrophotometer (Beckman Instruments, Schaumburg, IL, USA) and comparing those values against a glucose standard curve. Digestible starch (DS) was determined by subtracting (free glucose $\times 0.9$ ) from (total glucose/original sample weight) present in the supernatant after $15 \mathrm{~h}$ of digestion. The 0.9 value used in the calculation of DS is a correction factor for the difference in weight between a free glucose (FG) unit and a glucose residue in starch. Because the measurement of glucose was used to determine starch content, the correction factor was needed. Total starch (TS) content of samples was determined using the method of Thivend et al. [23] with amyloglucosidase. Resistant starch was calculated by subtracting [DS $+(\mathrm{FG} \times 0.9)]$ from TS. The released glucose value corresponds to the amount of glucose resulting from hydrolytic starch digestion that is available for absorption in vivo. Compositional data were not analyzed using statistical methods because accuracy was ensured by adequate replication, with acceptance of mean values that were within $5 \%$ of each other.

\section{Results}

The proximate, starch, essential amino acid (EAA), non-essential amino acid, and mineral concentrations of all samples are listed in Tables 1-5 respectively. All values are expressed on a dry matter basis (DMB). A visual representation of the CP, AHF, TDF, ash, and nitrogen free extract (NFE) fractions of all samples are listed in Figure 1. 
Table 1. Chemical composition of whole grain, processed grain, grain coproduct, and other carbohydrate sources.

\begin{tabular}{|c|c|c|c|c|c|c|c|c|}
\hline \multirow{3}{*}{ Item } & \multicolumn{8}{|c|}{$\% \mathrm{DM}^{1}$ Basis } \\
\hline & \multirow[b]{2}{*}{ DM, \% } & \multirow[b]{2}{*}{ OM } & \multicolumn{6}{|c|}{ Total Dietary Fiber } \\
\hline & & & $\mathrm{CP}$ & AHF & TDF & IDF & SDF & GE, $\mathrm{kcal} / \mathrm{g} \mathrm{DM}$ \\
\hline \multicolumn{9}{|c|}{ Barley category (Hordeum vulgare L.) } \\
\hline Barley flake & 91.8 & 97.8 & 14.5 & 8.5 & 10.8 & 6.5 & 4.3 & 4.8 \\
\hline Cut barley & 89.8 & 98.1 & 11.6 & 2.7 & 15.5 & 10.7 & 4.8 & 4.6 \\
\hline Ground pearled barley & 90.5 & 97.7 & 11.4 & 3.0 & 15.9 & 11.5 & 4.5 & 4.4 \\
\hline Malted barley & 95.6 & 97.3 & 13.5 & 3.2 & 42.1 & 22.9 & 19.2 & 4.8 \\
\hline Pearled barley flakes & 90.5 & 98.0 & 10.9 & 2.8 & 15.9 & 10.4 & 5.4 & 4.6 \\
\hline Steamed rolled barley & 90.3 & 97.4 & 11.3 & 3.1 & 23.1 & 19.3 & 3.9 & 4.5 \\
\hline Whole pearled barley & 89.2 & 97.9 & 11.3 & 2.9 & 13.4 & 8.6 & 4.8 & 4.7 \\
\hline \multicolumn{9}{|c|}{ Oat category (Avena sativa L.) } \\
\hline Groats & 90.5 & 97.6 & 12.1 & 7.4 & 9.9 & 7.9 & 2.0 & 4.7 \\
\hline Ground steamed groats & 91.6 & 98.0 & 12.4 & 8.0 & 10.9 & 9.9 & 1.1 & 4.8 \\
\hline Instant oats & 91.9 & 97.7 & 13.4 & 8.2 & 10.7 & 9.7 & 1.0 & 4.8 \\
\hline Oat bran \#1 & 91.2 & 97.4 & 13.9 & 8.4 & 11.0 & 7.3 & 3.7 & 4.9 \\
\hline Oat bran \#2 & 91.9 & 97.6 & 13.8 & 8.4 & 11.0 & 8.8 & 2.1 & 4.9 \\
\hline Oat fiber & 95.9 & 94.3 & 1.7 & 1.6 & 85.2 & 80.9 & 4.3 & 4.4 \\
\hline Oat flour & 91.9 & 98.0 & 13.6 & 8.1 & 10.4 & 6.0 & 4.4 & 4.8 \\
\hline Oatmeal (ground) & 92.2 & 97.6 & 13.6 & 8.8 & 10.2 & 7.8 & 2.4 & 4.9 \\
\hline Quick oats & 92.3 & 97.1 & 12.4 & 7.5 & 8.8 & 7.7 & 1.1 & 4.8 \\
\hline Regular rolled oats & 91.8 & 97.6 & 13.1 & 8.0 & 7.5 & 6.3 & 1.1 & 4.8 \\
\hline Steamed rolled oat groats & 90.7 & 97.6 & 13.8 & 7.5 & 6.6 & 3.4 & 3.2 & 4.8 \\
\hline Steel cut groats & 90.4 & 97.6 & 13.2 & 7.9 & 11.1 & 8.5 & 2.6 & 4.9 \\
\hline \multicolumn{9}{|c|}{ Rice category (Oryza sativa L.) } \\
\hline Brown rice & 89.3 & 97.4 & 10.3 & 4.6 & 9.7 & 8.8 & 0.9 & 4.4 \\
\hline Defatted rice bran & 90.6 & 84.3 & 17.0 & 4.4 & 24.2 & 22.5 & 1.7 & 4.3 \\
\hline Polished rice & 88.7 & 98.7 & 8.3 & 1.8 & 2.2 & 1.9 & 0.2 & 4.4 \\
\hline Rice flour & 92.4 & 96.1 & 8.9 & 1.5 & 8.6 & 5.6 & 3.0 & 4.1 \\
\hline \multicolumn{9}{|c|}{ Miscellaneous cereal grains and other carbohydrate sources } \\
\hline Canary grass seed & 93.4 & 93.8 & 19.7 & 7.3 & 20.8 & 17.6 & 3.1 & 4.5 \\
\hline Conventional whole millet & 92.9 & 96.1 & 10.4 & 5.0 & 15.3 & 13.9 & 1.4 & 4.3 \\
\hline Conventional hulled millet & 89.0 & 97.9 & 12.3 & 5.7 & 7.0 & 3.0 & 3.9 & 4.8 \\
\hline Conventional quinoa & 92.8 & 97.1 & 13.6 & 6.2 & 19.9 & 18.9 & 1.0 & 4.6 \\
\hline Organic spelt hull pellets & 89.2 & 95.4 & 10.2 & 3.2 & 44.0 & 39.0 & 5.0 & 4.5 \\
\hline Potato flake & 92.0 & 95.5 & 9.7 & 1.3 & 7.0 & 2.8 & 4.2 & 4.3 \\
\hline Sorghum & 88.6 & 98.3 & 10.4 & 4.3 & 12.5 & 9.9 & 2.7 & 4.5 \\
\hline Whole wheat & 89.3 & 97.7 & 12.2 & 2.6 & 13.8 & 13.2 & 0.6 & 4.5 \\
\hline Whole yellow corn & 88.7 & 98.6 & 7.1 & 5.1 & 13.5 & 12.1 & 1.4 & 4.6 \\
\hline
\end{tabular}

${ }^{1} \mathrm{DM}=$ dry matter; $\mathrm{OM}=$ organic matter; $\mathrm{CP}=$ crude protein; $\mathrm{AHF}$ = acid hydrolyzed fat; TDF = total dietary fiber; $\mathrm{IDF}=$ insoluble dietary fiber; $\mathrm{SDF}=$ soluble dietary fiber; $\mathrm{GE}=$ gross energy. 
Table 2. Total starch and starch fractions of whole grain, processed grain, grain coproduct, and other carbohydrate sources.

\begin{tabular}{|c|c|c|c|c|c|c|}
\hline \multirow{3}{*}{ Item } & \multicolumn{6}{|c|}{$\%$ DM Basis } \\
\hline & \multirow{2}{*}{ FG $^{1}$} & \multirow{2}{*}{ TS } & \multirow{2}{*}{$\begin{array}{c}\text { TS } \\
\text { (w/o FG) }\end{array}$} & \multirow{2}{*}{ DS } & DS & \multirow{2}{*}{ RS } \\
\hline & & & & & (w/o FG) & \\
\hline \multicolumn{7}{|c|}{ Barley category (Hordeum vulgare L.) } \\
\hline Barley flake & 0.08 & 67.7 & 67.6 & 63.9 & 63.8 & 3.8 \\
\hline Cut barley & 0.13 & 74.2 & 74.1 & 67.0 & 66.9 & 7.2 \\
\hline Ground pearled barley & 0.09 & 73.1 & 73.0 & 63.7 & 63.6 & 9.4 \\
\hline Malted barley & 0.09 & 16.2 & 16.1 & 11.4 & 11.3 & 4.8 \\
\hline Pearled barley flakes & 0.08 & 73.8 & 73.8 & 65.7 & 65.6 & 8.1 \\
\hline Steamed rolled barley & 0.08 & 67.7 & 67.6 & 61.9 & 61.9 & 5.7 \\
\hline Whole pearled barley & 0.11 & 72.3 & 72.2 & 64.9 & 64.8 & 7.4 \\
\hline \multicolumn{7}{|c|}{ Oat category (Avena sativa L.) } \\
\hline Groats & 0.10 & 73.4 & 73.3 & 69.5 & 69.4 & 3.9 \\
\hline Ground steamed groats & 0.07 & 71.9 & 71.8 & 67.6 & 67.6 & 4.3 \\
\hline Instant oats & 0.05 & 69.5 & 69.4 & 64.4 & 64.4 & 5.1 \\
\hline Oat bran \#1 & 0.07 & 65.3 & 65.2 & 64.3 & 64.3 & 0.9 \\
\hline Oat bran \#2 & 0.06 & 67.4 & 67.4 & 61.7 & 61.6 & 5.8 \\
\hline Oat fiber & 0.08 & 8.5 & 8.5 & 6.9 & 6.8 & 1.7 \\
\hline Oat flour & 0.06 & 69.2 & 69.2 & 65.6 & 65.5 & 3.7 \\
\hline Oatmeal (ground) & 0.07 & 66.2 & 66.2 & 61.8 & 61.7 & 4.4 \\
\hline Quick oats & 0.06 & 73.5 & 73.4 & 67.2 & 67.1 & 6.3 \\
\hline Regular rolled oats & 0.05 & 68.7 & 68.7 & 65.0 & 65.0 & 3.7 \\
\hline Steamed rolled oat groats & 0.06 & 71.2 & 71.1 & 66.1 & 66.0 & 5.1 \\
\hline Steel cut groats & 0.06 & 68.4 & 68.3 & 68.2 & 68.2 & 0.1 \\
\hline \multicolumn{7}{|c|}{ Rice category (Oryza sativa L.) } \\
\hline Brown rice & 0.16 & 77.4 & 77.2 & 66.8 & 66.7 & 10.6 \\
\hline Defatted rice bran & 0.06 & 34.2 & 34.2 & 29.8 & 29.7 & 4.4 \\
\hline Polished rice & 0.06 & 88.0 & 87.9 & 74.7 & 74.7 & 13.2 \\
\hline Rice flour & 0.48 & 73.6 & 73.2 & 66.8 & 66.4 & 6.3 \\
\hline \multicolumn{7}{|c|}{ Miscellaneous cereal grains and other carbohydrate sources } \\
\hline Canary grass seed & 0.17 & 49.7 & 49.5 & 47.3 & 47.1 & 2.4 \\
\hline Conventional whole millet & 0.14 & 64.9 & 64.7 & 61.1 & 61.0 & 3.8 \\
\hline Conventional hulled millet & 0.08 & 73.5 & 73.5 & 66.6 & 66.5 & 7.0 \\
\hline Conventional quinoa & 1.31 & 55.7 & 54.5 & 53.3 & 52.2 & 2.4 \\
\hline Organic spelt hull pellets & 0.16 & 42.0 & 41.9 & 38.3 & 38.2 & 3.7 \\
\hline Potato flake & 0.49 & 73.2 & 72.8 & 66.9 & 66.5 & 6.3 \\
\hline Sorghum & 0.20 & 70.5 & 70.4 & 63.3 & 63.1 & 7.2 \\
\hline Whole wheat & 0.19 & 68.7 & 68.6 & 62.2 & 62.0 & 6.4 \\
\hline Whole yellow corn & 0.24 & 65.0 & 64.8 & 65.6 & 65.3 & 0.0 \\
\hline
\end{tabular}

${ }^{1} \mathrm{FG}=$ free glucose; TS = total starch; DS = digestible starch; RS = resistant starch. 
Table 3. Essential amino acid (EAA) composition ${ }^{1}$ of whole grain, processed grain, grain coproduct, and other carbohydrate sources.

\begin{tabular}{|c|c|c|c|c|c|c|c|c|c|c|c|}
\hline \multirow{2}{*}{ Item } & \multicolumn{10}{|c|}{ Essential Amino Acids (\% DM Basis) } & \multirow{2}{*}{$\begin{array}{l}\text { Total } \\
\text { EAA }\end{array}$} \\
\hline & $\operatorname{Arg}^{2}$ & His & Ile & Leu & Lys & Met & Phe & Thr & Trp & Val & \\
\hline \multicolumn{12}{|c|}{ Barley category (Hordeum vulgare L.) } \\
\hline Barley flake & 0.94 & 0.32 & 0.57 & 1.11 & 0.66 & 0.27 & 0.77 & 0.52 & 0.14 & 0.76 & 6.06 \\
\hline Cut barley & 0.51 & 0.23 & 0.41 & 0.80 & 0.42 & 0.19 & 0.61 & 0.39 & 0.11 & 0.56 & 4.23 \\
\hline Ground pearled barley & 0.53 & 0.24 & 0.42 & 0.82 & 0.42 & 0.20 & 0.63 & 0.40 & 0.11 & 0.56 & 4.33 \\
\hline Malted barley & 0.01 & 0.05 & 0.30 & 0.67 & 0.06 & 0.10 & 0.49 & 0.05 & 0.04 & 0.47 & 2.24 \\
\hline Pearled barley flakes & 0.55 & 0.25 & 0.41 & 0.80 & 0.45 & 0.18 & 0.62 & 0.40 & 0.10 & 0.56 & 4.32 \\
\hline Steamed rolled barley & 0.49 & 0.23 & 0.42 & 0.80 & 0.41 & 0.20 & 0.61 & 0.39 & 0.11 & 0.55 & 4.21 \\
\hline Whole pearled parley & 0.58 & 0.25 & 0.42 & 0.82 & 0.48 & 0.20 & 0.62 & 0.42 & 0.10 & 0.59 & 4.48 \\
\hline \multicolumn{12}{|c|}{ Oat category (Avena sativa L.) } \\
\hline Groats & 0.83 & 0.27 & 0.49 & 0.96 & 0.57 & 0.23 & 0.67 & 0.48 & 0.14 & 0.65 & 5.29 \\
\hline Ground steamed groats & 0.79 & 0.27 & 0.48 & 0.93 & 0.55 & 0.20 & 0.65 & 0.42 & 0.15 & 0.64 & 5.08 \\
\hline Instant oats & 0.72 & 0.24 & 0.45 & 0.86 & 0.50 & 0.21 & 0.61 & 0.40 & 0.14 & 0.58 & 4.71 \\
\hline Oat bran \#1 & 0.74 & 0.26 & 0.47 & 0.90 & 0.53 & 0.22 & 0.63 & 0.41 & 0.17 & 0.61 & 4.94 \\
\hline Oat bran \#2 & 0.83 & 0.27 & 0.48 & 0.97 & 0.61 & 0.21 & 0.67 & 0.47 & 0.16 & 0.66 & 5.33 \\
\hline Oat fiber & 0.86 & 0.28 & 0.49 & 0.97 & 0.64 & 0.22 & 0.68 & 0.48 & 0.17 & 0.66 & 5.45 \\
\hline Oat flour & 0.07 & 0.02 & 0.07 & 0.15 & 0.07 & 0.03 & 0.08 & 0.07 & $<0.04$ & 0.09 & 0.65 \\
\hline Oatmeal (ground) & 0.81 & 0.27 & 0.49 & 0.96 & 0.57 & 0.21 & 0.67 & 0.45 & 0.16 & 0.65 & 5.24 \\
\hline Quick oats & 0.69 & 0.24 & 0.43 & 0.84 & 0.51 & 0.18 & 0.60 & 0.39 & 0.15 & 0.56 & 4.59 \\
\hline Regular rolled oats & 0.71 & 0.25 & 0.45 & 0.88 & 0.51 & 0.22 & 0.62 & 0.40 & 0.16 & 0.59 & 4.79 \\
\hline Steamed rolled oat groats & 0.76 & 0.25 & 0.49 & 0.95 & 0.50 & 0.22 & 0.67 & 0.43 & 0.14 & 0.63 & 5.04 \\
\hline Steel cut groats & 0.74 & 0.25 & 0.45 & 0.90 & 0.53 & 0.19 & 0.63 & 0.42 & 0.15 & 0.60 & 4.86 \\
\hline \multicolumn{12}{|c|}{ Rice category (Oryza sativa L.) } \\
\hline Brown rice & 0.79 & 0.26 & 0.43 & 0.84 & 0.44 & 0.24 & 0.54 & 0.38 & 0.07 & 0.60 & 4.59 \\
\hline Defatted rice bran & 1.38 & 0.47 & 0.62 & 1.23 & 0.88 & 0.34 & 0.77 & 0.66 & 0.20 & 0.96 & 7.51 \\
\hline Polished rice & 0.62 & 0.20 & 0.36 & 0.70 & 0.35 & 0.20 & 0.45 & 0.28 & 0.08 & 0.50 & 3.74 \\
\hline Rice flour & 0.44 & 0.15 & 0.31 & 0.45 & 0.49 & 0.15 & 0.38 & 0.30 & 0.10 & 0.48 & 3.25 \\
\hline \multicolumn{12}{|c|}{ Miscellaneous cereal grains and other carbohydrate sources } \\
\hline Canary grass seed & 1.05 & 0.37 & 0.80 & 1.45 & 0.42 & 0.26 & 1.18 & 0.46 & 0.32 & 0.86 & 7.17 \\
\hline Conventional whole millet & 1.07 & 0.38 & 0.54 & 0.87 & 0.79 & 0.26 & 0.56 & 0.45 & 0.13 & 0.62 & 5.67 \\
\hline Conventional hulled millet & 0.40 & 0.24 & 0.51 & 1.49 & 0.20 & 0.36 & 0.69 & 0.39 & 0.08 & 0.61 & 4.97 \\
\hline Conventional quinoa & 0.51 & 0.17 & 0.33 & 0.49 & 0.51 & 0.14 & 0.38 & 0.32 & 0.11 & 0.49 & 3.45 \\
\hline Organic spelt hull pellets & 0.40 & 0.23 & 0.41 & 1.23 & 0.29 & 0.19 & 0.52 & 0.36 & 0.06 & 0.52 & 4.21 \\
\hline Potato flake & 0.46 & 0.24 & 0.40 & 0.76 & 0.36 & 0.18 & 0.52 & 0.35 & 0.10 & 0.49 & 3.86 \\
\hline Sorghum & 0.33 & 0.22 & 0.44 & 1.32 & 0.18 & 0.27 & 0.60 & 0.30 & 0.08 & 0.54 & 4.28 \\
\hline Whole wheat & 0.55 & 0.27 & 0.44 & 0.84 & 0.40 & 0.20 & 0.57 & 0.39 & 0.18 & 0.54 & 4.38 \\
\hline Whole yellow corn & 0.36 & 0.21 & 0.26 & 0.78 & 0.29 & 0.15 & 0.35 & 0.25 & 0.06 & 0.35 & 3.06 \\
\hline
\end{tabular}

${ }^{1}$ AOAC, 2006; method 982.30E. ${ }^{2}$ Arg = arginine; His = histidine; Ile = isoleucine; Leu = leucine; Lys = lysine; Met = methionine; Phe = phenylalanine; Thr = threonine; Trp = tryptophan; Val = valine 
Table 4. Nonessential amino acid (NEAA) composition ${ }^{1}$ of whole grain, processed grain, grain coproduct, and other carbohydrate sources.

\begin{tabular}{|c|c|c|c|c|c|c|c|c|c|c|c|c|c|}
\hline \multirow{2}{*}{ Item } & \multicolumn{12}{|c|}{ Nonessential Amino Acids (\% DM Basis) } & \multirow{2}{*}{$\begin{array}{l}\text { Total } \\
\text { NEAA }\end{array}$} \\
\hline & Ala ${ }^{2}$ & Asp & Cys & Glu & Gly & Hyl & Hyp & Orn & Pro & Ser & Tau & Tyr & \\
\hline \multicolumn{14}{|c|}{ Barley category (Hordeum vulgare L.) } \\
\hline Cut barley & 0.45 & 0.68 & 0.23 & 2.71 & 0.43 & 0.03 & 0.01 & 0.00 & 1.24 & 0.45 & 0.17 & 0.26 & 6.66 \\
\hline Ground pearled Barley & 0.43 & 0.67 & 0.24 & 2.85 & 0.44 & 0.02 & 0.01 & 0.00 & 1.28 & 0.46 & 0.17 & 0.29 & 6.86 \\
\hline Malted barley & 0.42 & 0.46 & 0.03 & 1.97 & 0.33 & 0.05 & 0.02 & 0.06 & 0.86 & 0.02 & 0.14 & 0.24 & 4.60 \\
\hline Whole pearled barley & 0.48 & 0.73 & 0.23 & 2.68 & 0.49 & 0.02 & 0.01 & 0.00 & 1.21 & 0.47 & 0.16 & 0.29 & 6.77 \\
\hline \multicolumn{14}{|c|}{ Oat category (Avena sativa L.) } \\
\hline Groats & 0.61 & 1.05 & 0.42 & 2.63 & 0.66 & 0.02 & 0.00 & 0.01 & 0.67 & 0.62 & 0.17 & 0.41 & 7.27 \\
\hline Ground steamed groats & 0.57 & 0.95 & 0.38 & 2.57 & 0.63 & 0.01 & 0.01 & 0.01 & 0.64 & 0.56 & 0.17 & 0.37 & 6.87 \\
\hline Instant oats & 0.51 & 0.92 & 0.35 & 2.47 & 0.55 & 0.02 & 0.00 & 0.01 & 0.61 & 0.57 & 0.14 & 0.35 & 6.50 \\
\hline Oatmeal (ground) & 0.58 & 1.01 & 0.40 & 2.69 & 0.62 & 0.01 & 0.01 & 0.01 & 0.65 & 0.61 & 0.17 & 0.38 & 7.14 \\
\hline Quick oats & 0.50 & 0.90 & 0.36 & 2.41 & 0.54 & 0.01 & 0.01 & 0.01 & 0.60 & 0.55 & 0.15 & 0.29 & 6.33 \\
\hline Regular rolled oats & 0.51 & 0.92 & 0.35 & 2.51 & 0.56 & 0.01 & 0.00 & 0.01 & 0.61 & 0.56 & 0.19 & 0.32 & 6.55 \\
\hline Steamed rolled oat groats & 0.56 & 0.97 & 0.36 & 2.83 & 0.63 & 0.02 & 0.00 & 0.01 & 0.75 & 0.60 & 0.17 & 0.39 & 7.29 \\
\hline Steel cut groats & 0.55 & 0.95 & 0.39 & 2.52 & 0.60 & 0.02 & 0.01 & 0.01 & 0.62 & 0.58 & 0.15 & 0.37 & 6.77 \\
\hline \multicolumn{14}{|c|}{ Rice category (Oryza sativa L.) } \\
\hline Brown rice & 0.58 & 0.93 & 0.21 & 1.75 & 0.48 & 0.01 & 0.01 & 0.01 & 0.45 & 0.48 & 0.17 & 0.28 & 5.36 \\
\hline Defatted rice bran & 1.06 & 1.53 & 0.33 & 2.42 & 0.94 & 0.04 & 0.06 & 0.01 & 0.76 & 0.74 & 0.14 & 0.44 & 8.47 \\
\hline Polished rice & 0.46 & 0.76 & 0.18 & 1.48 & 0.38 & 0.01 & 0.00 & 0.01 & 0.37 & 0.39 & 0.12 & 0.20 & 4.36 \\
\hline Rice flour & 0.32 & 1.86 & 0.12 & 1.67 & 0.26 & 0.31 & 0.02 & 0.01 & 0.29 & 0.29 & 0.17 & 0.26 & 5.58 \\
\hline Whole wheat & 0.45 & 0.66 & 0.27 & 3.50 & 0.50 & 0.01 & 0.01 & 0.01 & 1.20 & 0.57 & 0.17 & 0.27 & 7.62 \\
\hline Whole yellow corn & 0.52 & 0.50 & 0.16 & 1.24 & 0.34 & 0.02 & 0.03 & 0.00 & 0.62 & 0.33 & 0.10 & 0.17 & 4.03 \\
\hline
\end{tabular}

${ }^{1}$ AOAC, 2006; method 982.30E. ${ }^{2}$ Ala = alanine; Asp = aspartic acid; Cys = cysteine; Glu = glutamic acid; Gly = glycine; Hyl = hydroxylysine; Hyp = hydroxyproline; Orn = ornithine; Pro = proline; Ser = serine; Tau $=$ taurine $;$ Tyr $=$ tyrosine . 
Table 5. Mineral composition of whole grain, processed grain, grain coproduct, and other carbohydrate sources.

\begin{tabular}{|c|c|c|c|c|c|c|c|}
\hline Item & $\mathrm{Ca}^{1,2}$ & $\mathrm{Cl}^{1,3}$ & $\mathrm{Mg}^{1,2}$ & $\mathbf{P}^{1,2}$ & $\mathrm{~K}^{1,4}$ & $\mathrm{Na}^{1,4}$ & $S^{1,4}$ \\
\hline \multicolumn{8}{|c|}{ 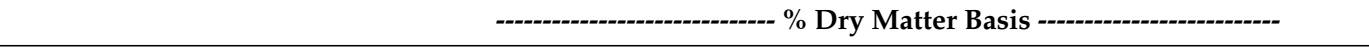 } \\
\hline \multicolumn{8}{|c|}{ Barley category (Hordeum vulgare L.) } \\
\hline Barley flake & 0.04 & $<0.10$ & 0.11 & 0.33 & 0.30 & $<0.10$ & 0.10 \\
\hline Cut barley & 0.03 & $<0.10$ & 0.10 & 0.27 & 0.30 & $<0.10$ & $<0.10$ \\
\hline Ground pearled barley & 0.03 & $<0.10$ & 0.10 & 0.28 & 0.30 & $<0.10$ & 0.10 \\
\hline Malted barley & 0.05 & $<0.10$ & 0.15 & 0.40 & 0.40 & $<0.10$ & 0.10 \\
\hline Pearled barley flakes & 0.03 & $<0.10$ & 0.10 & 0.30 & 0.30 & $<0.10$ & 0.10 \\
\hline Steamed rolled barley & 0.04 & $<0.10$ & 0.11 & 0.31 & 0.40 & $<0.10$ & 0.10 \\
\hline Whole pearled barley & 0.03 & $<0.10$ & 0.11 & 0.31 & 0.30 & $<0.10$ & $<0.10$ \\
\hline \multicolumn{8}{|c|}{ Oat category (Avena sativa $L)}$. \\
\hline Groats & 0.04 & $<0.10$ & 0.10 & 0.33 & 0.30 & $<0.10$ & 0.10 \\
\hline Ground steamed groats & 0.03 & $<0.10$ & 0.07 & 0.24 & 0.30 & $<0.10$ & 0.10 \\
\hline Instant oats & 0.03 & $<0.10$ & 0.09 & 0.27 & 0.30 & $<0.10$ & $<0.10$ \\
\hline Oat bran \#1 & 0.03 & $<0.10$ & 0.10 & 0.32 & 0.30 & $<0.10$ & 0.10 \\
\hline Oat bran \#2 & 0.04 & $<0.10$ & 0.11 & 0.36 & 0.40 & $<0.10$ & 0.10 \\
\hline Oat fiber & 0.08 & $<0.10$ & 0.06 & 0.04 & 0.50 & $<0.10$ & $<0.10$ \\
\hline Oat flour & 0.04 & $<0.10$ & 0.11 & 0.32 & 0.30 & $<0.10$ & 0.10 \\
\hline Oatmeal (ground) & 0.04 & $<0.10$ & 0.11 & 0.35 & 0.30 & $<0.10$ & $<0.10$ \\
\hline Quick oats & 0.03 & $<0.10$ & 0.06 & 0.25 & 0.30 & $<0.10$ & $<0.10$ \\
\hline Regular rolled oats & 0.03 & $<0.10$ & 0.09 & 0.27 & 0.30 & $<0.10$ & $<0.10$ \\
\hline Steamed rolled oat groats & 0.03 & $<0.10$ & 0.08 & 0.25 & 0.30 & $<0.10$ & 0.10 \\
\hline Steel cut groats & 0.03 & $<0.10$ & 0.08 & 0.25 & 0.30 & $<0.10$ & $<0.10$ \\
\hline \multicolumn{8}{|c|}{ Rice category (Oryza sativa L.) } \\
\hline Brown rice & 0.01 & $<0.10$ & 0.13 & 0.37 & 0.30 & $<0.10$ & $<0.10$ \\
\hline Defatted rice bran & 2.22 & $<0.10$ & 0.88 & 2.03 & 1.50 & $<0.10$ & $<0.10$ \\
\hline Polished rice & 0.00 & $<0.10$ & 0.03 & 0.14 & 0.10 & $<0.10$ & $<0.10$ \\
\hline Rice flour & 0.05 & $<0.10$ & 0.09 & 0.23 & 1.30 & $<0.10$ & $<0.10$ \\
\hline \multicolumn{8}{|c|}{ Miscellaneous cereal grains and other carbohydrate sources } \\
\hline Canary grass seed & 0.03 & $<0.10$ & 0.17 & 0.49 & 0.40 & $<0.10$ & 0.20 \\
\hline Conventional whole millet & 0.01 & $<0.10$ & 0.12 & 0.27 & 0.20 & $<0.10$ & $<0.10$ \\
\hline Conventional hulled millet & 0.01 & $<0.10$ & 0.17 & 0.37 & 0.30 & $<0.10$ & 0.10 \\
\hline Conventional quinoa & 0.05 & $<0.10$ & 0.20 & 0.45 & 0.80 & $<0.10$ & 0.10 \\
\hline Organic spelt hull pellets & 0.07 & $<0.10$ & 0.15 & 0.40 & 0.40 & $<0.10$ & 0.10 \\
\hline Potato flake & 0.03 & 0.20 & 0.09 & 0.21 & 1.70 & $<0.10$ & $<0.10$ \\
\hline Sorghum & 0.02 & $<0.10$ & 0.18 & 0.36 & 0.40 & $<0.10$ & 0.10 \\
\hline Whole wheat & 0.03 & $<0.10$ & 0.09 & 0.30 & 0.30 & $<0.10$ & $<0.10$ \\
\hline Whole yellow corn & 0.00 & $<0.10$ & 0.09 & 0.26 & 0.30 & $<0.10$ & $<0.10$ \\
\hline
\end{tabular}

${ }^{1} \mathrm{Ca}=$ Calcium; $\mathrm{Cl}$ = Chloride; $\mathrm{Mg}=$ Magnesium; $\mathrm{P}=$ Phosphorus; $\mathrm{K}=$ Potassium; $\mathrm{Na}=$ Sodium; $\mathrm{S}=$ Sulfur. ${ }^{2}$ AOAC, 2006; method 985.01. ${ }^{3}$ AOAC, 2006; method 943.01. ${ }^{4}$ AOAC, 2006; method 956.01. 


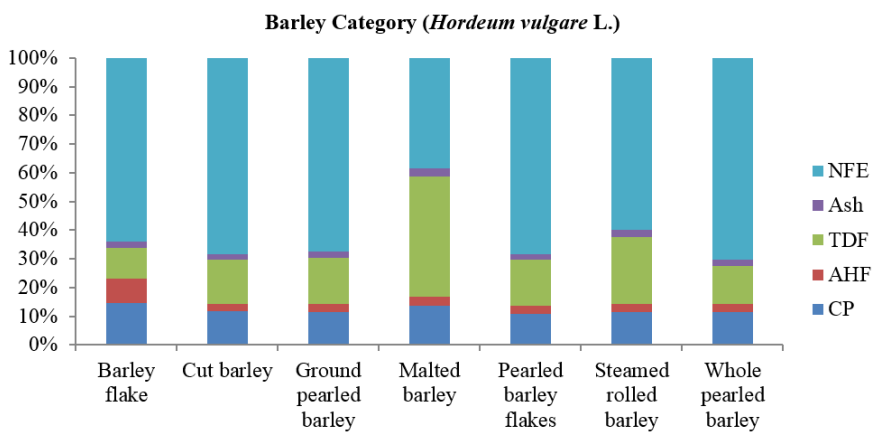

(a)

Oat Category (Avena sativa L.)

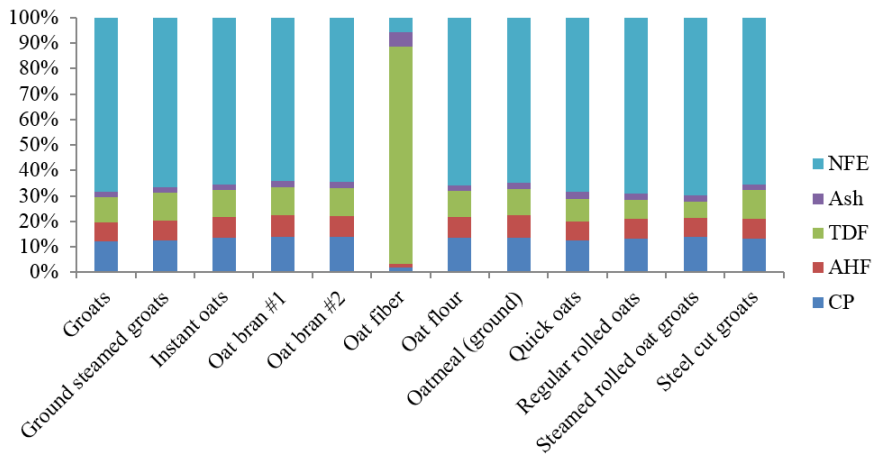

(b)

Rice Category (Oryza sativa L.)

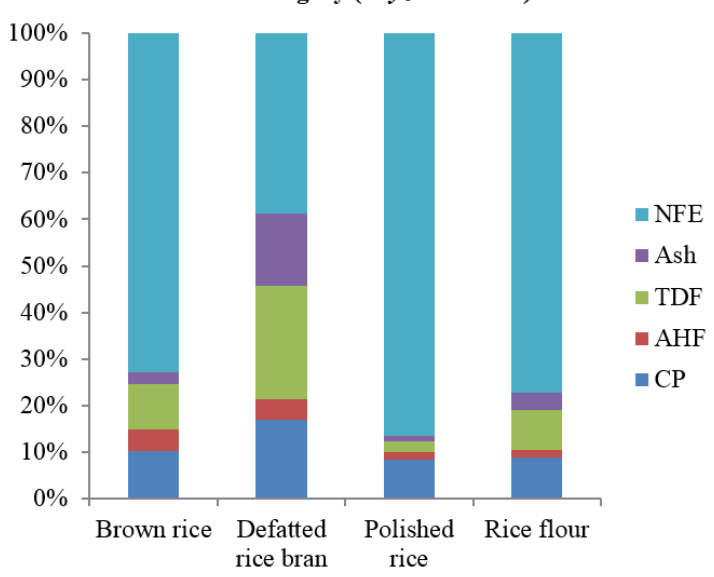

(c)

Miscellaneous Carbohydrate Sources

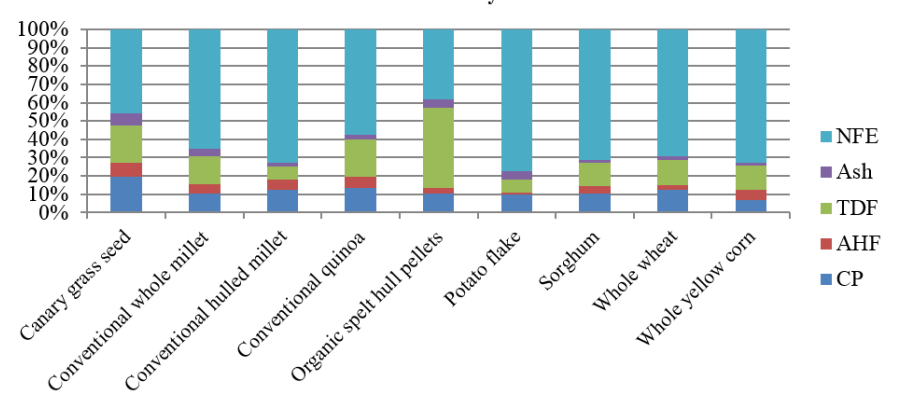

(d)

Figure 1. Multi-colored stacked bar graphs represent the crude protein (CP), acid hydrolyzed fat (AHF), total dietary fiber (TDF), ash, and nitrogen free extract (NFE) fractions of whole grain, processed grain, grain coproduct, and other carbohydrate sources. The ingredients are organized into 4 main categories: (a) barley category (Hordeum vulgare L.); (b) oat category (Avena sativa L.); (c) rice category (Oryza sativa L.); and (d) miscellaneous carbohydrate sources. 


\subsection{Barley Category (Hordeum vulgare L.)}

Samples in the barley category had DM concentrations that ranged from $89.2 \%$ (whole pearled barley) to $95.6 \%$ (malted barley). Organic matter concentrations were very similar among all barley-based ingredients (97.3\% to $98.1 \%)$. Crude protein had a greater range (10.9\% to $14.5 \%)$, but was still fairly similar among ingredients. Despite the similarities in DM, OM, and CP, a couple barley-based ingredients were unique in regards to their total lipid, TDF, and starch content. Barley flake, for instance, contained 2.5 times more fat (8.5\%) than any other barley-based ingredient. Total dietary fiber was quite variable as well. While most ingredients had TDF concentrations below 16\%, steam rolled barley (23.1\%) and malted barley (42.1\%) had higher concentrations, with malted barley having approximately half of its fiber in the soluble form, which is usually the most fermentable form. Gross energy, starch, AA, and mineral concentrations were not greatly different among most barley-based ingredients. The most notable difference in terms of starch content was the malted barley, which had low total starch and the other fractions due to its higher TDF concentration.

\subsection{Oat Category (Avenal sativa L.)}

All samples in the oat category had high and similar DM (>90.4\%) and OM (94.3\%) concentrations. Although most oat-based ingredients had a CP and total lipid concentration above $12 \%$ and $7 \%$, respectively, oat fiber had a very low $\mathrm{CP}(1.7 \%)$ and lipid $(1.6 \%)$ concentrations. In contrast, oat fiber had a very high TDF concentration (85.2\%), while all others contained less than $11 \%$. The low lipid content led to a low gross energy content $(4.44 \mathrm{kcal} / \mathrm{g})$ of oat fiber, with all others having more than $4.7 \mathrm{kcal} / \mathrm{g}$. Not surprisingly, oat fiber also had low starch fractions, often containing 5 to 10 times lower concentrations than the other oat-based ingredients.

\subsection{Rice Category (Oryza sativa L.)}

All samples in the rice category had high and similar DM (>88.7\%) concentrations. Organic matter and CP concentrations were more variable among the rice ingredients and ranged from $84.3 \%$ to $98.7 \%$ and $8.3 \%$ to $17.0 \%$, respectively. Gross energy concentrations were not different among the rice ingredients $(\sim 4 \mathrm{kcal} / \mathrm{g})$ and all samples had total lipid concentrations $<5 \%$. Defatted rice bran had more TDF (24.2\% TDF) than the other rice ingredients tested, most of which was insoluble (22.5\% IDF). Polished rice contained the highest total starch (87.9\%). All sample had similar AA concentrations. Among the rice ingredients tested, defatted rice bran was the most rich in many of the minerals tested, including $\mathrm{Ca}, \mathrm{P}, \mathrm{K}$, and $\mathrm{Mg}$.

\subsection{Miscellaneous Cereal Grains and Other Carbohydrate Sources}

All samples in the miscellaneous category had high and similar DM (>88.6\%) and OM (93.8\%) concentrations. Crude protein was more variable among the ingredients tested and ranged from $7.1 \%$ to $19.7 \%$. Gross energy concentrations were not different among the miscellaneous ingredients $(\sim 4 \mathrm{kcal} / \mathrm{g})$ and all samples had low total lipid concentrations that were all $<10 \%$. Both quinoa and canary grass seed had high TDF concentrations (20\% TDF), most of which was insoluble (18\% IDF). Organic spelt hull pellets also had a high TDF concentration (44\% TDF) of which the majority was insoluble (39\% IDF). Conventional hulled millet contained the highest total starch (73.5\%) and sorghum contained the highest resistant starch (7.2\%). All samples had similar AA concentrations. Potato flakes were rich in $\mathrm{K}(1.70 \%)$.

\section{Discussion}

The objective of this study was to measure the proximate, starch, AA, and mineral composition of various whole grains, processed grains, and grain co-products that may be incorporated into pet foods and treats. Cereal grains and other carbohydrate sources are widely used in pet food formulations, but poorly studied. Therefore, a detailed compositional analysis of commonly used cereal grains in 
addition to the in vivo effects of feeding novel cereal grains to pets is greatly needed. This study, which focused on the compositional analysis, may not only be useful for pet food formulators, but may be used to design in vivo studies to compare palatability, nutrient digestibility, and/or effects on host health in the future.

Barley (Hordeum vulgare L.) is rich in fermentable and soluble fibers and is gaining interest from the pet food industry as a novel carbohydrate source. Barley is harvested with the hull attached and contains a high level of $\beta$-glucans. Steamed rolled barley has steam applied above the roller mill to decrease the production of fine particles and allows for a more uniform particle size [24-27]. Cut barley, also known as barley grits, is produced when barley kernels are cut into several small pieces. If cut barley comes from hulled or hulless barley, it is considered to be a whole grain, but cut barley from pearl barley is not [25-27]. Barley flakes are produced when steam is applied to whole grain barley kernels, then rolled and dried. Barley flakes have decreased cooking time because of the steam that had been applied and increased the surface area [25-28]. Whole pearled barley has the hull removed and has been polished to remove some or the entire outer bran layer. Pearled barley can be tan or white and is not technically considered a whole grain, but is more nutritious than other refined grains (e.g., polished rice) due to its high concentrations of $\beta$-glucans and fiber distributed throughout the kernel. Pearled barley cooks more rapidly than whole grain barley because both the tough outer bran layer and hull have been polished off, and is the most common type of barley sold [25,27,29]. Pearled barley flakes are produced the same way as barley flakes, but from pearled barley kernels [25-28]. Ground pearled barley is pearled barley kernels that have been ground to a meal form for use as flour [25-27]. Malted barley requires several more processing steps: (1) initial steeping, where whole kernels are soaked to achieve a moisture content of $42 \%-46 \%$ (approximately $48-52 \mathrm{~h}$ ); (2) germination, where hydrolytic enzymes are synthesized by the aleurone cells and scutellum, and are eventually secreted into the starchy endosperm of the soaked barley kernel, promoting endosperm modification (typically performed at $13-16{ }^{\circ} \mathrm{C}$ for $8-10$ days); and (3) kilning, drying process to cease germination and preserve the malt (typically dried to an approximate final moisture of $2 \%-3 \%$ ) [24]. Malted barley then can be used for brewing, distilling, or malt vinegar production $[25,30]$. Evaluation of the inclusion of barley into extruded dog diets is limited, but one study concluded that $40 \%$ extruded barley into a common basal diet (corn, wheat, and animal fat) resulted in decreased fecal dry matter or looser stools when fed to dogs [31]. The TDF of barley may have contributed to the looser stools observed in those dogs. The barley evaluated in the current set of ingredients had high TDF, about half of which was soluble fiber. More research is needed to fully evaluate the use of barley or barley fractions in extruded diets for dogs, but these data suggest it may be a good source of dietary fiber.

Oats (Avena sativa L.) are not the most common cereal grain used in pet foods, but the use of oats is growing in popularity due to their nutrient profile and lack of pet exposure, which is especially important in hypersensitivity or elimination diets [32]. Oats are harvested with the hull attached, which is often removed prior to consumption. Oat bran and germ are rarely removed, however. Like barley, oats are high in $\beta$-glucans and other constituents, such as carbohydrates, proteins, avenanthramides, tocols, lipids, alkaloids, flavonoids, saponins, and sterols [33]. Oat groats are cleaned oat kernels that have had their inedible hull removed and take the longest to cook [34]. Steel cut groats are groats that have been cut into 2-3 pieces with a sharp metal blade. They are often referred to as Irish oatmeal and cook faster than whole oat groats $[35,36]$. Steamed rolled oat groats are produced by applying steam to whole oat groats while being rolled into flakes $[35,36]$. Ground steamed groats are rolled oat groats that have been ground to a meal form [35]. Regular rolled oats are often referred to as old fashioned oats. They are created when oat groats are steamed and rolled into flakes. Regular rolled oat groats are thinner than steamed rolled oat groats, but thicker than quick or instant oats. This process also helps to preserve the healthy lipids in the oat $[35,36]$. Oatmeal is ground rolled oats $[35,36]$. Quick and instant oats are oat flakes that have been cut, then rolled thinner and steamed longer, ultimately resulting in a change in texture and decreased cooking time $[35,36]$. Oat bran is produced when whole oat groats are passed through several rollers that flatten the kernels. The bran is separated from the 
flour and sifted to be further separated, resulting in oat bran and de-branned oat flour [37]. Oat fiber is produced from finely ground oat hulls [35,36]. Oat flour is produced from whole groats sent to a stone or hammer mill that then can be used for baking or thickening of soups and stews $[35,36]$. Many of the aforementioned oat ingredients are readily available, cost-effective, and nutritionally consistent carbohydrate options for pet food manufacturers [32]. While not all of the health benefits identified when humans consume oats directly translate to dogs and cats, the $\beta$-glucans and dietary fiber found in oats may be beneficial in decreasing the prevalence of obesity and diabetes in pets.

Rice (Oryza sativa L.) is commonly used in pet foods due to its relative low cost and ease of procurement. Rice is commonly consumed worldwide and is easily digested. Rice is harvested with the hull attached and is known as paddy rice. Brown rice is a rice kernel that has had the hull removed and is rich in B vitamins and minerals [38]. Polished rice has had both the hull and bran removed, therefore making it less nutritious than brown rice [38]. Rice bran is produced from the outer layers of the harvested kernel and is rich in fiber, vitamins, and minerals [38,39], with the majority of rice bran produced being incorporated into diets for animals [40]. The high content of fiber and minerals found in rice bran may provide an alternative fiber or mineral source for canine diets. From a processing perspective, it is important to note that heat treatment can inactivate the lipase activity in the rice seed coat, greatly decreasing the shelf-life of this ingredient. This can lead to increased oxidation, rancidity, and undesirable odors and flavors [41]. Furthermore, the phytochemicals in rice bran may provide dogs and cats with additional health benefits, but these compounds were not evaluated in the current dataset. Rice flour is produced from broken white rice kernels that have been finely milled $[38,42]$ and provides a flour option for gluten-free products.

Several cereal grains and carbohydrate sources also were evaluated, many of which are not currently used in the pet food industry. Canary grass seed (or annual canarygrass; Phalaris canariensis L.) is a grain crop that is produced similarly to oat and wheat and is most commonly used as birdfeed [43]. Whole yellow corn (Zea mays L.) is the largest size cereal grain produced and is the most produced grain worldwide, for both food and non-edible products [35]. Millet (proso variety; Panicum miliaceum L.) is a group of several small seeded grains. In the U.S., it is most typically used as birdfeed and livestock feed, but millet is consumed by humans in India, Africa, China, Japan, and parts of Europe [44]. Whole millet is cleaned and sized with the hull still attached, whereas hulled millet has had the hull removed. Potato flakes were first introduced as a means to increase shelf-life of potatoes. To produce potato flakes, potatoes are first peeled, trimmed, and sliced. Potato slices then are cooked at a low temperature $\left(150-160^{\circ} \mathrm{C}\right)$ for $20 \mathrm{~min}$, known as a pre-cook step. Next, they are cooled to halt the cooking process and as a means to decrease the stickiness of the starchy vegetable. The final cooking step occurs in a steam cooker for approximately 15-60 min. Finally, the potatoes are dried in a single-drum drier and broken into flakes [45]. Quinoa (Chenopodium quinoa Willd.) is a tiny, round, highly nutritious pseudocereal, often light-colored, but also can be red, purple, or black [46,47]. Sorghum grain (milo; Sorghum bicolor L.) is a hearty grain that can survive under conditions that other grains would not (e.g., drought). Some sorghum varieties contain polyphenols or other pigments/tannins that contain anti-nutritional factors. In the U.S., it is commonly used for livestock feed, but is a great source of fiber and easily incorporated into human and pet food [48,49]. Spelt (Triticum spelta L.) is an ancient subspecies of wheat. Spelt is often used as an alternative feed grain, but can also be used as a food grain once the hulls are removed. Spelt hull pellets are comprised of spelt hulls that have been ground and formed into pellets [50,51]. Wheat (Triticum aestivum L., or bread wheat) is the most common grain used in breads, cakes and pastries, and other grain foods. Wheat contains a protein, gluten, which contributes to the elasticity of bread dough [52]. The demand for alternative carbohydrate sources is ever-growing with the increased demand for "no-corn" and "no-wheat" diets for dogs and cats. Therefore, developing a more robust database of the composition of alternative carbohydrate sources will be beneficial as the use of these alternative carbohydrates increases.

Of the whole ingredients tested (i.e., whole pearled barley, groats, brown rice, canary grass seed, conventional quinoa, conventional whole millet, sorghum, whole wheat, and whole yellow corn), $\mathrm{CP}$ 
values were lowest in whole yellow corn and highest in canary grass seed. One interesting observation was the total essential amino acid concentration of quinoa, typically advertised for its high protein and essential amino acid content. However, in our evaluation, it was not one of the highest in either crude protein or essential amino acids. Fat values were lowest in whole wheat and highest in groats. Total dietary fiber concentrations were lowest in brown rice and highest in canary grass seed. Nitrogen free extract fractions were lowest in canary grass seed and highest in whole yellow corn. The demand to use less processed, more whole, natural ingredients continues to increase in both the human and pet food industries. It is possible that the use of whole grains may affect processing of the diet by contributing to increased breaking or lack of formation of the food or treat. A potential limitation of this study was the possibility that a portion of RS could have been decreased through the methodology performed to obtain the compositional data (i.e., if total starch samples were allowed to cool below $55^{\circ} \mathrm{C}$, then reheated to $55^{\circ} \mathrm{C}$ for $24 \mathrm{~h}$, resulting in altered content of RS). This may make application or formulation using these ingredients difficult because raw ingredients were tested and it has been well documented that RS concentration and the concentrations of other nutrients can be impacted by pet food processing (e.g., extrusion; retort; baking) [15,53,54]. Furthermore, the inclusion of whole ingredients may affect the digestibility and bioavailability of nutrients in the animal, but in vivo testing is necessary to determine these effects.

\section{Conclusions}

In conclusion, the whole grains studied herein varied when compared to their respective fractions (i.e., barley flake vs. malted barley vs. steamed rolled barley) as well as compared to other grains (i.e., barley flake $v$ s. brown rice $v$ s. canary grass seed). The samples evaluated in this study included a variety of carbohydrate and fiber sources including: whole grains, grain fractions, processed grains, and other carbohydrate sources, such as potato flakes. Based on our analyses, we believe the most interesting ingredients for future research include oat fiber, malted barley, rice bran, canary grass seed, and barley flake. These ingredients are valuable because of their total dietary fiber content, insoluble:soluble fiber ratio, and AA profile. Compositional information generated from this study provides a framework for many grains, some of which do not have much data available. Further investigation of these ingredients for their effects in vivo is justified. These ingredients may not only beneficially alter indices of gastrointestinal health, but they may impact pet food formulation by potentially decreasing the amount of animal proteins needed, which can be costly from a formulation standpoint. Although grains are often called "fillers" and grain-free diets are increasingly popular with pet owners, our data demonstrate that these ingredients may contribute both as a readily available energy source and a source of dietary fiber, RS, EAA, and macrominerals for pet diets.

Acknowledgments: The Nutro Company provided funding for this research through The Nutro Company Natural Pet Nutrition Fellowship.

Author Contributions: The project was designed by authors Beloshapka, Buff, and Swanson. Beloshapka conducted the analyses. All authors contributed to data interpretation. Beloshapka wrote the manuscript and all authors revised and approved the final manuscript.

Conflicts of Interest: The authors declare no conflict of interest.

\section{References}

1. American Association of Cereal Chemists International (AACC). Whole Grain Task Force and Definition. 2009. Available online: http://www.aaccnet.org/definitions/wholegrain.asp (accessed on 17 March 2014).

2. Okarter, N.; Liu, R.H. Health benefits of whole grain phytochemicals. Crit. Rev. Food Sci. Nutr. 2010, 50, 193-208. [CrossRef] [PubMed]

3. De Moura, F.F.; Lewis, K.D.; Falk, M.C. Applying the FDA definition of whole grains to the evidence for cardiovascular disease health claims. J. Nutr. 2009, 139, 2220S-2226S. [CrossRef] [PubMed]

4. Anderson, J.W. Whole grains protect against atherosclerotic cardiovascular disease. Proc. Nutr. Soc. 2003, 62, 135-142. [CrossRef] [PubMed] 
5. Slavin, J. Whole grains and human health. Nutr. Res. Rev. 2004, 17, 99-110. [CrossRef] [PubMed]

6. Englyst, H.N.; Kingman, S.M.; Cummings, J.H. Classification and measurement of nutritionally important starch fractions. Eur. J. Clin. Nutr. 1992, 46, S33-S50. [PubMed]

7. Sajilata, M.G.; Singhal, R.S.; Kulkarni, P.R. Resistant starch: A review. Compr. Rev. Food Sci. Food Saf. 2006, 5, 1-17. [CrossRef]

8. Case, L.P.; Carey, D.P.; Hirakawa, D.A. Canine and Feline Nutrition: A Resource for Companion Animal Professionals; Mosby, Inc.: St. Louis, MO, USA, 1995.

9. Laflamme, D.P.; Abood, S.K.; Fascetti, A.J.; Fleeman, L.M.; Freeman, L.M.; Michel, K.E.; Bauer, C.; Kemp, B.L.E.; Van Doren, J.R.; Willoughby, K.N. Pet feeding practices of dog and cat owners in the United States and Austrailia. J. Am. Vet. Med. Assoc. 2008, 232, 687-694. [CrossRef] [PubMed]

10. Phillips-Donaldson, D. Why dry petfood rules the world. Petfood Ind. 2013, 55, 6.

11. Bradshaw, J.W.S. The evolutionary basis for the feeding behaviour of domestic dogs (Canis familiaris) and cats (Felis catus). J. Nutr. 2006, 136, 1927S-1931S. [PubMed]

12. Stevens, E.J.; Armstrong, K.W.; Bezar, H.J.; Griffin, W.B.; Hampton, J.G. Fodder Oats: A World Overview; Plant Production and Protection Series No. 33; Suttie, J.M., Reynolds, S.G., Eds.; Food and Agriculture Organization of the United Nations: Rome, Italy, 2004.

13. Food and Agriculture Organization of the United Nations (FAO). World Food Situation: FAO Cereal Supply and Demand Brief. 2014. Available online: www.fao.org/worldfoodsituation/csdb/en (accessed on 17 March 2014).

14. Beaton, L. Grain-free petfood: A top trend in the U.S. pet market. Petfood Ind. 2014, 56, 68-70.

15. Hernot, D.C.; Boileau, T.W.; Bauer, L.L.; Swanson, K.S.; Fahey, G.C., Jr. In vitro digestion characteristics of unprocessed and processed whole grains and their components. J. Agric. Food Chem. 2008, 56, 10721-10726. [CrossRef] [PubMed]

16. Muyonga, J.H.; Andabati, B.; Ssepuuya, G. Effect of heat processing on selected grain amaranth physicochemical properties. Food Sci. Nutr. 2014, 2, 9-16. [CrossRef] [PubMed]

17. Association of Official Analytical Chemists (AOAC). Official Methods of Analysi, 17th ed.; Association of Official Analytical Chemists: Gaithersburg, MD, USA, 2006.

18. American Association of Cereal Chemists (AACC). Approved Methods, 8th ed.; American Association of Cereal Chemists: St Paul, MN, USA, 1983.

19. Budde, E.F. The determination of fat in baked biscuit type of dog foods. J. Assoc. Off. Agric. Chem. 1952, 35, 799-805.

20. Prosky, L.; Asp, N.G.; Schweizer, T.F.; De Vries, J.W.; Fruda, I. Determination of insoluble and soluble dietary fiber in foods and food products: Collaborative study. J. AOAC Int. 1992, 75, 360-367.

21. Muir, J.G.; O'Dea, K. Measurement of resistant starch: Factors affecting the amount of starch escaping digestion in vitro. Am. J. Clin. Nutr. 1992, 56, 123-127. [PubMed]

22. Muir, J.G.; O'Dea, K. Validation of an in vitro assay for predicting the amount of starch that escapes digestion in the small intestine of humans. Am. J. Clin. Nutr. 1993, 57, 540-546. [PubMed]

23. Thivend, P.; Christiane, M.; Guilbot, A. Determination of starch with glucoamylase. Methods Carbohydr. Chem. 1972, 6, 100-105.

24. Hironaka, R.; Kimura, N.; Kozub, G.C. Influence of feed particle size on rate and efficiency of gain, characteristics of rumen fluid and rumen epithelium, and numbers of rumen protozoa. Can. J. Anim. Sci. 1979, 589, 395-402. [CrossRef]

25. Bamforth, C.W.; Barclay, A.H.P. Malting technology and the uses of malt. In Barley Chemistry and Technology; MacGregor, A.W., Bhatty, R.S., Eds.; American Association of Cereal Chemists: St Paul, MN, USA, 1993; pp. 297-354.

26. Mathison, G.W. Effects of processing on the utilization of grain by cattle. Anim. Feed Sci. Technol. 1996, 58, 113-125. [CrossRef]

27. Dehghan-Banadaky, M.; Corbett, R.; Oba, M. Effects of barley grain processing on productivity of cattle. Anim. Feed Sci. Technol. 2007, 137, 1-24. [CrossRef]

28. Zinn, R.A. Influence of processing on the comparative feeding value of barley for feedlot cattle. J. Anim. Sci. 1993, 71, 3-10. [PubMed] 
29. Engstrom, D.F.; Mathison, G.W.; Goonewardene, L.A. Effect of $\beta$-glucan, starch, and fibre content and steam vs. dry rolling of barley grain on its degradability and utilization by steers. Anim. Feed Sci. Technol. 1992, 37, 33-46. [CrossRef]

30. Haraldsson, A.K.; Rimsten, L.; Alminger, M.L.; Andersson, R.; Andlid, T.; Åman, P.; Sandberg, A.S. Phytate content is reduced and $\beta$-glucanase activity suppressed in malted barley steeped with lactic acid at high temperature. J. Sci. Food Agric. 2004, 84, 653-662. [CrossRef]

31. Groner, T.; Pfeffer, E. Digestibility of organic matter and digestible energy in single ingredients of extruded dog feeds and their effect on fecal dry matter concentration and consistency. J. Anim. Physiol. Anim. Nutr. 1997, 77, 214-220. [CrossRef]

32. Aldrich, G. Oats: Cheerios for pets? Petfood Ind. 2006, 49, 40-41.

33. Singh, R.; De, S.; Belkheir, A. Avena sativa (oat), a potential neutraceutical and therapeutic agent: An overview. Crit. Rev. Food Sci. Nutr. 2013, 53, 126-144. [CrossRef] [PubMed]

34. Welch, R.; Brown, J.; Leggett, J. Interspecific and intraspecific variation in grain and groat characteristics of wild oat (Avena) species: Very high groat (1-3), (1-4)-b-D-glucan in an Avena atlantica genotype. J. Cereal Sci. 2000, 31, 273-279. [CrossRef]

35. Owens, F.N.; Secrist, D.S.; Hill, W.J.; Gill, D.R. The effect of grain source and grain processing on performance of feedlot cattle: A review. J. Anim. Sci. 1997, 75, 868-879. [PubMed]

36. Winfield, K.; Hall, M.; Paynter, B. Milling Oat and Feed Oat Quality: What Are the Differences; Bulletin 4703; Department of Agriculture and Food, Government of Western Austrailia: South Perth, WA, Australia, 2007; pp. 10-12.

37. Sadiq Butt, M.; Tahir-Nadeem, M.; Khan, M.K.; Shabir, R.; Butt, M.S. Oat: Unique among the cereals. Eur. J. Nutr. 2008, 47, 68-79. [CrossRef] [PubMed]

38. Shams-Ud-Din, M.D.; Bhattacharaya, K.R. On the meaning of the degree of milling of rice. J. Food Technol. 1978, 13, 99-105. [CrossRef]

39. Saunders, R.M. The properties of rice bran as a foodstuff. Cereal Foods World 1990, 35, 632-636.

40. Kahlon, T.S. Rice Bran: Production, Composition, Functionality and Food Applications, Physiological Benefits. In Fiber Ingredients: Food Applications and Health Benefits; Cho, S.S., Samuel, P., Eds.; CRC Press; Taylor \& Francis Group: Boca Raton, FL, USA, 2009; pp. 305-322.

41. Ryan, E.P. Bioactive food components and health properties of rice bran. J. Am. Vet. Med. Assoc. 2011, 238, 593-600. [CrossRef] [PubMed]

42. Hasjim, J.; Li, E.; Dhital, S. Milling of rice grains: Effects of starch/flour structures on gelatinization and pasting properties. Carbohydr. Polym. 2013, 92, 862-690. [CrossRef] [PubMed]

43. Putnam, D.H.; Oelke, E.A.; Oplinger, E.S.; Doll, J.D.; Peters, J.B. Annual Canarygrass. Alternative Field Crops Manual. University of Wisconsin-Extension, University of Minnesota: Center for Alternative Plant and Animal Products, and the Minnesota Extension Service. 1990. Available online: http://www. hort.purdue.edu/newcrop/afcm/cangrass.html (accessed on 6 March 2014).

44. Oelke, E.A.; Oplinger, E.S.; Putnam, D.H.; Durgan, B.R.; Doll, J.D.; Undersander, D.J. Millets. Alternative Field Crops Manual. University of Wisconsin-Extension, University of Minnesota: Center for Alternative Plant and Animal Products, and the Minnesota Extension Service. 1990. Available online: http:/ / www.hort.purdue.edu/newcrop/afcm/millet.html (accessed on 6 March 2014).

45. Ginsberg, J. Development of Dehydration Processes: Potato Flakes; American Chemical Society: Washington, DC, USA, 2007.

46. Oelke, E.A.; Putnam, D.H.; Teynor, T.M.; Oplinger, E.S. Quinoa. Alternative Field Crops Manual. University of Wisconsin-Extension, University of Minnesota: Center for Alternative Plant and Animal Products, and the Minnesota Extension Service. 1992. Available online: http://www.hort.purdue. edu/newcrop/afcm/quinoa.html (accessed on 6 March 2014).

47. Abugoch, L.E. Quinoa (Chenopodium quinoa Willd.) composition, chemistry, nutritional, and functional properties. Adv. Food Nutr. Res. 2009, 58,1-31.

48. Carter, P.R.; Hicks, D.R.; Oplinger, E.S.; Doll, J.D.; Bundy, L.G.; Schuler, R.T.; Holmes, B.J. Grain sorghum (milo). Alternative Field Crops Manual. University of Wisconsin-Extension, University of Minnesota: Center for Alternative Plant and Animal Products, and the Minnesota Extension Service. 1989. Available online: http:/ /www.hort.purdue.edu/newcrop/afcm/sorghum.html (accessed on 6 March 2014). 
49. Twomey, L.N.; Pethick, D.W.; Rowe, J.B.; Choct, M.; Pluske, J.R.; Brown, W.; Laviste, M.C. The use of sorghum and corn as alternatives to rice in dog foods. J. Nutr. 2002, 132, 1704S-1705S. [PubMed]

50. Oplinger, E.S.; Oelke, E.A.; Kaminski, A.R.; Kelling, K.A.; Doll, J.D.; Durgan, B.R.; Schuler, R.T. Spelt. Alternative Field Crops Manual. University of Wisconsin-Extension, University of Minnesota: Center for Alternative Plant and Animal Products, and the Minnesota Extension Service. 1990. Available online: http:/ / www.hort.purdue.edu/newcrop/afcm/spelt.html (accessed on 6 March 2014).

51. Neeson, R. Organic spelt production. Industry and Investment NSW. 2011. Available online: www.industry. nsw.gov.au/publications (accessed on 6 March 2014).

52. Shewry, P.R.; Hawkesford, M.J.; Piironen, V.; Lampi, A.; Gebruers, K.; Boros, D.; Andersson, A.A.M.; Åman, P.; Rakszegi, M.; Bedo, Z.; et al. Natural variation in grain composition of wheat and related cereals. J. Agric. Food Chem. 2013, 61, 8295-8303. [CrossRef] [PubMed]

53. Murray, S.M.; Flickinger, E.A.; Patil, A.R.; Merchen, N.R.; Brent, J.L., Jr.; Fahey, G.C., Jr. In vitro fermentation characteristics of native and processed cereal grains and potato starch using ileal chyme from dogs. J. Anim. Sci. 2001, 79, 435-444. [PubMed]

54. Dust, J.M.; Gajda, A.M.; Flickinger, E.A.; Burkhalter, T.M.; Merchen, N.R.; Fahey, G.C., Jr. Extrusion conditions affect chemical composition and in vitro digestion of select food ingredients. J. Agric. Food Chem. 2004, 52, 2989-2996. [CrossRef] [PubMed]

(C) 2016 by the authors; licensee MDPI, Basel, Switzerland. This article is an open access article distributed under the terms and conditions of the Creative Commons by Attribution (CC-BY) license (http://creativecommons.org/licenses/by/4.0/). 